\title{
Tackling the Photovoltaic Integration Challenge in the Distribution Network with Deferrable Load
}

\author{
Enxin Yao, Pedram Samadi, Vincent W.S. Wong, and Robert Schober \\ Department of Electrical and Computer Engineering \\ University of British Columbia, Vancouver, Canada \\ e-mail: $\{$ enxinyao, psamadi, vincentw, rschober\}@ece.ubc.ca
}

\begin{abstract}
In recent years, there is an increasing deployment of photovoltaic (PV) units and energy storage systems (ESSs) in households. When the energy generated by PV units is greater than the aggregate load of households and the capacity of ESS, there will be a reverse energy flow from households to the substation. When the reverse energy flow exceeds a certain threshold, it will cause a voltage rise problem, which is a challenge for PV units to be effectively integrated with the distribution network. In this paper, we propose an energy consumption scheduling algorithm, which shifts the deferrable load (e.g., washing machines, dryers) from peak hours (e.g., 7 pm - 10 pm) to high solar radiation hours (e.g., $10 \mathrm{am}-2 \mathrm{pm}$ ) in order to jointly shave the peak load and reduce the reverse energy flow. We formulate the energy consumption scheduling problem as a stochastic optimization problem to capture the uncertainty of the amount of PV output power. The objective of our algorithm is to minimize the electricity bill for the household users which have PV units and ESS installed. We use inclining block rate (IBR) pricing and time of use (TOU) pricing to encourage users to shift their load. Numerical results show that our proposed algorithm can avoid the voltage rise problem and reduce the peak-to-average ratio (PAR) in the aggregate load.
\end{abstract}

\section{INTRODUCTION}

In recent years, the use of photovoltaic (PV) systems and energy storage systems (ESSs) in households has proliferated. Many utility companies use a net metering program to encourage households to install roof-top PV units, which helps reduce their electricity bills and the pollution caused by fossil fuels [1]. However, in those areas with a large portion of households equipped with PV units and ESS, the power may flow from households to the substation in the distribution network during high solar radiation hours (e.g., 10 am - 2 $\mathrm{pm}$ ). The traditional voltage control strategy of the distribution network typically assumes that the power only flows from the substation to households. When this reverse energy flow from the households to the substation is greater than a certain threshold, it will cause the voltage rise problem [2]. This problem is a challenge for a large number of PV units to be integrated effectively with the distribution network.

Previous works have shown that some of the household load (e.g., water heater) as well as ESS (e.g., battery systems) can be used to reduce the reverse energy flow during high solar radiation hours and mitigate the voltage rise problem [3], [4]. The work in [3] proposes the activation of additional heating, ventilation, and air conditioning (HVAC) load whenever voltage rise is detected. A control algorithm for the ESS to reduce the reverse energy flow is proposed in [4]. However, in order to shape users' load to absorb the reverse energy flow, it is important to encourage households to participate in the load shifting by providing economic incentives to them. Moreover, it is necessary to equip households with automatic energy consumption scheduling devices to shape the load profile while satisfying the demand requirements of the users.

Most of the existing works in the literature on demand side management (DSM) [5]-[7] focus on reducing the peak load. Peak shaving reduces the energy generation cost at peak hours and plant investment. Dynamic pricing schemes, e.g., time of use (TOU) pricing, are typically used to encourage households to shift their load. The price-based DSM schemes presented in [5]-[7] can be adopted to tackle other problems besides peak shaving, such as the PV integration challenge in the distribution network.

We now summarize some of the related works on DSM for distributed renewable generation. The work in [8] proposes a game-theoretic approach to jointly schedule the load, distributed renewable generation, and ESS. A distributed scheduling algorithm is proposed in [9], which minimizes the cost for the utility company and the user payments. The work in [10] proposes a joint load scheduling and ESS energy management algorithm. A load scheduling algorithm is proposed in [11] to minimize the energy cost for residential homes. However, distributed renewable generation is typically installed in the distribution network, which is constrained by the direction of the power flow from the substation to the households. It is important to investigate how to use DSM to reduce the reverse energy flow to the distribution network, especially for areas with a high penetration of distributed renewable generation, such as PV.

In this paper, we propose an energy consumption scheduling algorithm to mitigate the adverse impact (e.g., voltage rise) of PV integration on the distribution network by reducing the reverse energy flow. The contributions are as follows:

- We propose an energy consumption scheduling algorithm, which shifts deferrable load (e.g., water heaters, washing machines, and dryers) from the peak hours to high solar radiation hours. The proposed algorithm reduces both the peak load and the reverse energy flow. We adopt both inclining block rate (IBR) pricing tariffs and TOU pricing to encourage load shifting.

- We formulate the energy consumption scheduling prob- 


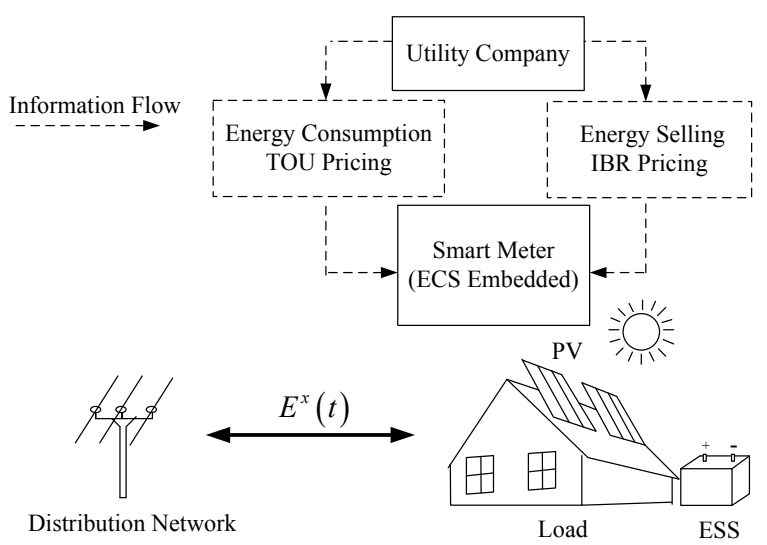

Fig. 1. The schematic diagram of the proposed energy consumption scheduling scheme.

lem as a stochastic optimization problem in order to model the uncertainty of the PV output power. We tackle the problem by using the sample average approximation (SAA) technique.

- We compare our proposed algorithm with another algorithm from [3]. Simulation results show that our proposed algorithm provides a lower household electricity bill. Results also show that our algorithm can avoid the voltage rise problem caused by $\mathrm{PV}$ integration and reduce the peak-to-average ratio (PAR) of the aggregate load.

The rest of the paper is organized as follows. The system model is introduced in Section II. In Section III, we present the problem formulation and the energy consumption scheduling algorithm. Numerical results are presented in Section IV, and the paper is concluded in Section V.

\section{SySTEM MOdEL}

A block diagram of the proposed energy consumption scheduling scheme for a household equipped with a PV unit and ESS is shown in Fig. 1. The power flows from the distribution network to the load if the power generated by the PV unit is not sufficient to support the load. The direction of the power flow is reversed if the power generated by the PV unit is higher than the load. Each household is equipped with an energy consumption scheduler (ECS) [5], which is embedded in the household's smart meter. The ECS retrieves the pricing information from the utility company and determines the operational schedule of the deferrable load. Examples of such deferrable load include washing machines, dryers, and water heaters. Some other loads such as TV and computers are must-run load [7] because their energy consumption cannot be shifted. We also consider ESS in our model, e.g., the battery systems owned by the household. We denote the sets of deferrable load, must-run load, and ESS by $\mathcal{S}_{d}, \mathcal{S}_{m}$, and $\mathcal{S}_{s}$, respectively.

We denote the set of operating time slots (e.g., hours) under consideration as $\mathcal{T} \triangleq\{1, \ldots, T\}$. For $i \in \mathcal{S}_{d}, j \in \mathcal{S}_{m}, l \in \mathcal{S}_{s}$, we denote the energy consumption of the $i$ th deferrable load, the energy consumption of the $j$ th must-run load, the charged or discharged energy of the $l$ th ESS at time slot $t \in \mathcal{T}$, as

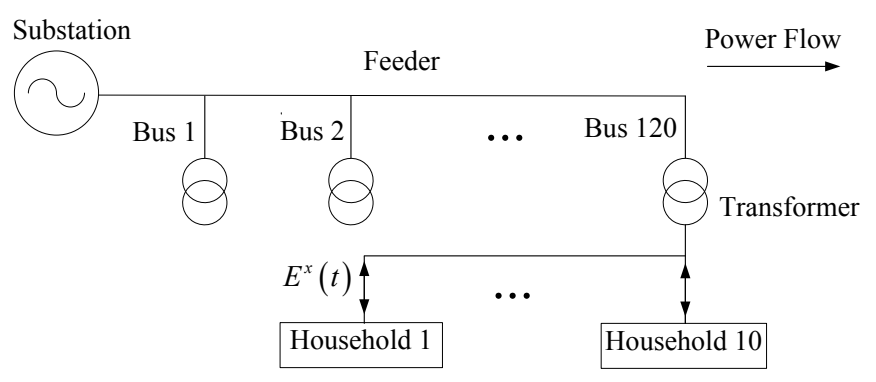

Fig. 2. The schematic diagram of a feeder in the area with high PV penetration. The feeder is connected with several buses. Each bus is connected with multiple households equipped with PV unit and ESS. We focus on the energy consumption scheduling of a single household (e.g., household 1).

$E_{i}^{d}(t), E_{j}^{m}(t)$, and $E_{l}^{s}(t)$, respectively. We have $E_{l}^{s}(t)>0$ for charging and $E_{l}^{s}(t)<0$ for discharging. $E^{g}(t)$ represents the energy generated from PV unit at time slot $t$. We denote the reverse energy flow at time slot $t$ by $E^{x}(t)$. We have

$$
E^{x}(t)=E^{g}(t)-\sum_{i \in \mathcal{S}_{d}} E_{i}^{d}(t)-\sum_{j \in \mathcal{S}_{m}} E_{j}^{m}(t)-\sum_{l \in \mathcal{S}_{s}} E_{l}^{s}(t) .
$$

Most of the time, the PV output power is absorbed by the load and the ESS. During those time intervals, we have $E^{x}(t) \leq 0$. On the other hand, during the time interval when the power generated by the PV unit exceeds the aggregate load and the capacity of the ESS, we have $E^{x}(t)>0$.

The schematic diagram of a feeder in high PV penetration area is shown in Fig. 2. Multiple households equipped with PV units and ESS are connected with the feeder. When many of these households have positive reverse energy flow, the energy may flow back to the substation along the feeder, and cause the voltage rise problem. In this paper, we use the price-based energy consumption scheduling to tackle this problem. The pricing scheme encourages households to reduce the reverse energy flow by load shifting. Note that we focus on the energy consumption scheduling of a single household (e.g., household 1). The ECS in each household only controls its own load and ESS.

The inclining block rate (IBR) pricing scheme is currently adopted by some utility companies (e.g., Southern California Edison) to encourage households to conserve energy. A higher rate is charged for household energy consumption over a certain amount in the IBR pricing scheme. In this paper, we adopt the concept of IBR pricing scheme and use it for the household PV energy selling in the areas with high PV penetration. The households sell their extra energy at a higher price when the reverse energy flow to the distribution network is small. If the reverse energy flow exceeds a certain threshold, the excess portion beyond the threshold is sold at a lower price.

The IBR pricing scheme considered in this paper is illustrated in Fig. 3. We denote the TOU energy consumption price at time slot $t$ by $p_{e}(t) . p_{s}^{h}(t)$ and $p_{s}^{l}(t)$ represent the energy selling price at time slot $t$ below and above threshold $h$ (e.g., $2 \mathrm{kWh}$, respectively. We set $p_{e}(t) \geq p_{s}^{h}(t)>p_{s}^{l}(t)$ as an incentive for the household to reduce the reverse energy flow.

Let $\mathbf{e}_{t}=\left(E_{1}^{d}(t), \ldots, E_{\left|\mathcal{S}_{d}\right|}^{d}(t), E_{1}^{s}(t), \ldots, E_{\left|\mathcal{S}_{s}\right|}^{s}(t)\right)$ denote 


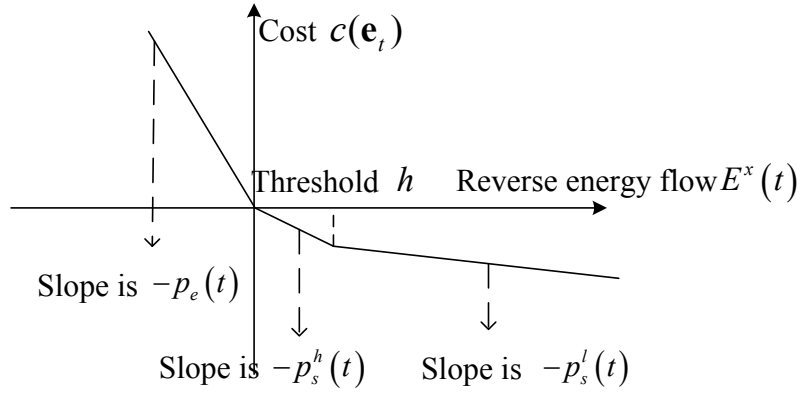

Fig. 3. The piecewise linear IBR pricing scheme for the household with PV generation.

the vector of energy consumption of the deferrable load and the charged or discharged energy of the ESS at time slot $t$, where $\left|\mathcal{S}_{d}\right|$ and $\left|\mathcal{S}_{s}\right|$ are the cardinality of sets $\mathcal{S}_{d}$ and $\mathcal{S}_{s}$, respectively. Note that household PV units are typically nondispatchable. We model both the PV and must-run load as non-controllable, i.e., the value of $E^{g}(t)$ is only determined by the environment (i.e., the weather). The ECS is only allowed to control $\mathbf{e}_{t}$. The user payment at time slot $t$ is written as

$$
c\left(\mathbf{e}_{t}\right)= \begin{cases}-p_{s}^{h}(t) h-p_{s}^{l}(t)\left(E^{x}(t)-h\right), & \text { if } E^{x}(t)>h, \\ -p_{s}^{h}(t) E^{x}(t), & \text { if } 0<E^{x}(t) \leq h, \\ p_{e}(t)\left|E^{x}(t)\right|, & \text { if } E^{x}(t) \leq 0,\end{cases}
$$

where $\left|E^{x}(t)\right|$ denotes the absolute value of $E^{x}(t)$. From equation (1) and Fig. 3, the cost function $c\left(\mathbf{e}_{t}\right)$ is a piecewise linear function of $E^{x}(t)$.

\section{ENERGY CONSUMPTION SCHEDULING WITH PV OUTPUT POWER UNCERTAINTY}

In this section, we consider the energy consumption scheduling problem for minimizing the expected payment of the user. We formulate the problem as a stochastic optimization problem [12]. Stochastic optimization is used to model the PV output power uncertainty. Note that the PV output power $E^{g}(t)$ is intermittent and hard to forecast precisely [13]. The ECS needs to determine $\mathbf{e}_{t}$ at the current time slot $t$, before the future PV outputs $E^{g}(t+1), \ldots, E^{g}(T)$ can be observed. In the next time slot $t+1$, the power generated from $\mathrm{PV}$ unit is revealed and the ECS needs to determine $\mathbf{e}_{t+1}$ without the knowledge of $E^{g}(t+2), \ldots, E^{g}(T)$. For the rest of the paper, we denote the the current time slot by $t . \tau$ represents an arbitrary time slot. We define $\mathcal{T}_{t} \triangleq\{t, \ldots, T\}$ as the set of time slots from the current time slot $t$ onwards.

\section{A. Nested Stochastic Formulation}

For $i \in \mathcal{S}_{d}$, we denote the amount of energy required by $i$ th deferrable load to complete its operation from the current time slot $t$ onwards by $E_{i}^{r}(t)$. For each time slot $t$, the energy requirement $E_{i}^{r}(t)$ is updated as

$$
E_{i}^{r}(t+1)=\max \left\{E_{i}^{r}(t)-E_{i}^{d}(t), 0\right\}, \forall i \in \mathcal{S}_{d} .
$$

We denote the maximum energy consumption of the $i$ th deferrable load within one time slot by $E_{i, d}^{\max } . E_{l, s}^{\max } \geq 0$ represents the energy charged in one time slot if the $l$ th ESS charges with the maximum power. $E_{l, s}^{\min } \leq 0$ is the energy discharged if the $l$ th ESS discharges with the maximum power. We denote the state of charge (SOC) of the lth ESS at time slot $\tau$ by $s_{l}(\tau)$. The battery capacity of the $l$ th ESS is denoted by $b_{l}, l \in \mathcal{S}_{s}$. At the current time slot $t$, the ECS determines $\mathbf{e}_{t}$ to minimize the cost from the current time slot $t$ onwards. The nested form of our stochastic problem is

$$
\underset{\mathbf{e}_{t} \in \chi_{t}}{\operatorname{minimize}} c\left(\mathbf{e}_{t}\right)+\mathbb{E}\left[\inf _{\mathbf{e}_{t+1} \in \chi_{t+1}} c\left(\mathbf{e}_{t+1}\right)+\cdots+\mathbb{E}\left[\inf _{\mathbf{e}_{T} \in \chi_{T}} c\left(\mathbf{e}_{T}\right)\right]\right],
$$

subject to $\sum_{\tau=t}^{T_{i}^{d}} E_{i}^{d}(\tau)=E_{i}^{r}(t), \forall i \in \mathcal{S}_{d}$,

where $T_{i}^{d}$ denotes the deadline by which the operation of deferrable load $i \in \mathcal{S}_{d}$ has to be finished, $\mathbb{E}$ denotes the expectation with respect to the uncertain PV output power in (4a). Note that the must-run load may also have uncertainty, depending on how accurate we can forecast the must-run load profile. The feasible set $\chi_{\tau}, \tau \in \mathcal{T}_{t}$ in (4a) can be written as

$$
\begin{aligned}
\chi_{\tau}=\left\{\mathbf{e}_{\tau} \mid\right. & 0 \leq E_{i}^{d}(\tau) \leq E_{i, d}^{\max }, \forall i \in \mathcal{S}_{d}, \\
& s_{l}(\tau)=s_{l}(\tau-1)+\frac{E_{l}^{s}(\tau)}{b_{l}}, \forall l \in \mathcal{S}_{s}, \\
& S^{\min } \leq s_{l}(\tau) \leq 1, \forall l \in \mathcal{S}_{s}, \\
& \left.E_{l, s}^{\min } \leq E_{l}^{s}(\tau) \leq E_{l, s}^{\max }, \forall l \in \mathcal{S}_{s}\right\}
\end{aligned}
$$

where $S^{\text {min }}$ is a threshold. The objective function (4a) has a nested form (i.e., multiple expectation $\mathbb{E}$ and infimum inf) to capture the process for the ECS to determine $\mathbf{e}_{\tau}$ dynamically at each time slot $\tau \in \mathcal{T}_{t}$. The constraint (4b) guarantees that each deferrable load completes its task before deadline.

Problem (4) is difficult to solve directly because the decision variables in different time slots are mutually dependent, as shown in constraint (4b). This dependency prevents using the conventional approach of taking expectations over each time slot independently. The computational complexity of directly solving the nested form stochastic optimization problem (4) grows exponentially with the time scale $\left|\mathcal{T}_{t}\right|$ (i.e., the cardinality of set $\mathcal{T}_{t}$ ) [12]. In order to reduce the complexity, we adopt the sample average approximation (SAA) technique [12] to solve problem (4). SAA technique generates scenarios for future unknown process and evaluates the objective function by averaging under several scenarios.

\section{B. Sample Average Approximation}

The random process for future PV output $\left(E^{g}(t+\right.$ $\left.1), \ldots, E^{g}(T)\right)$ is unknown for the ECS at the current time slot $t$, as the PV output power is intermittent and difficult to predict precisely. Suppose we have $K$ scenarios $\left\{\omega_{1}, \ldots, \omega_{K}\right\}$ of random process $\left(E^{g}(t+1), \ldots, E^{g}(T)\right)$. Each scenario is a possible realization of $\left(E^{g}(t+1), \ldots, E^{g}(T)\right)$. We define $\omega_{k} \triangleq\left(E_{k}^{g}(t+1), \ldots, E_{k}^{g}(T)\right)$, where $E_{k}^{g}(\tau), \tau \in \mathcal{T}_{t+1}$ is a possible value of $E^{g}(\tau)$. The scenarios $\left\{\omega_{1}, \ldots, \omega_{K}\right\}$ can be generated from the historical record of PV outputs. We define $\mathcal{K} \triangleq\{1, \ldots, K\}$ as the set of scenarios. 
Let $E_{i}^{d}\left(\tau, \omega_{k}\right), E_{j}^{m}\left(\tau, \omega_{k}\right), E_{l}^{s}\left(\tau, \omega_{k}\right), E^{x}\left(\tau, \omega_{k}\right), s_{l}\left(\tau, \omega_{k}\right)$, and $\mathbf{e}\left(\tau, \omega_{k}\right)$ denote the energy consumption of the $i$ th deferrable load, the energy consumption of the $j$ th must-run load, the charged or discharged energy of the lth ESS, the energy export, the SOC of the lth ESS, and the energy consumption vector at time slot $\tau$ under scenario $\omega_{k}$, respectively. We define vector $\mathbf{e}^{\max } \triangleq(\gamma, \ldots, \gamma) \in \mathbb{R}^{\left|\mathbf{e}_{\tau}\right|}$, where $\gamma$ is an arbitrarily large constant (e.g., $\left.\gamma=\sum_{i \in \mathcal{S}_{d}} E_{i, d}^{\max }+\sum_{l \in \mathcal{S}_{l}} E_{l, s}^{\max }\right)$. $\left|\mathbf{e}_{\tau}\right|$ is the cardinality of vector $\mathbf{e}_{\tau}$.

We estimate the expected user payment by averaging the user payments over different scenarios $\omega_{k}, \forall k \in \mathcal{K}$. Since the scenarios are generated by using historical data, we assign the same for each scenario. By using SAA technique, problem (5) becomes

$$
\begin{aligned}
& \underset{\substack{\mathbf{e}\left(\tau, \omega_{k}\right), \tau \in \mathcal{\mathcal { T } _ { t }}, \forall k \in \mathcal{K}}}{\operatorname{minimize}} \frac{1}{K} \sum_{k \in \mathcal{K}} \sum_{\tau \in \mathcal{T}_{t}} c\left(\mathbf{e}\left(\tau, \omega_{k}\right)\right) \\
& \text { subject to } \sum_{\tau=t}^{T_{i}^{d}} E_{i}^{d}\left(\tau, \omega_{k}\right)=E_{i}^{r}(t), \\
& \forall i \in \mathcal{S}_{d}, \forall k \in \mathcal{K}, \\
& 0 \leq E_{i}^{d}\left(\tau, \omega_{k}\right) \leq E_{i, d}^{\max }, \\
& \forall i \in \mathcal{S}_{d}, \forall \tau \in \mathcal{T}_{t}, \forall k \in \mathcal{K}, \\
& s_{l}\left(\tau, \omega_{k}\right)=s_{l}\left(\tau-1, \omega_{k}\right)+\frac{E_{l}^{s}\left(\tau, \omega_{k}\right)}{b_{l}}, \\
& \forall l \in \mathcal{S}_{s}, \forall \tau \in \mathcal{T}_{t}, \forall k \in \mathcal{K}, \\
& S^{\min } \leq s_{l}\left(\tau, \omega_{k}\right) \leq 1, \\
& \forall l \in \mathcal{S}_{s}, \forall \tau \in \mathcal{T}_{t}, \forall k \in \mathcal{K}, \\
& E_{l, s}^{\min } \leq E_{l}^{s}\left(\tau, \omega_{k}\right) \leq E_{l, s}^{\max }, \\
& \forall l \in \mathcal{S}_{s}, \forall \tau \in \mathcal{T}_{t}, \forall k \in \mathcal{K}, \\
& \mathbf{e}\left(\tau, \omega_{l}\right)-1_{\mathbf{d}_{\tau, \omega_{k}} \neq \mathbf{d}_{\tau, \omega_{l}}} \mathbf{e}^{\max } \preceq \mathbf{e}\left(\tau, \omega_{k}\right) \\
& \preceq \mathbf{e}\left(\tau, \omega_{l}\right)+1_{\mathbf{d}_{\tau, \omega_{k}} \neq \mathbf{d}_{\tau, \omega_{l}}} \mathbf{e}^{\max }, \\
& \forall \tau \in \mathcal{T}_{t+1}, \forall k, l \in \mathcal{K}, \\
& \mathbf{e}\left(t, \omega_{k}\right)=\mathbf{e}\left(t, \omega_{l}\right), \forall k, l \in \mathcal{K},
\end{aligned}
$$

where $\preceq$ in constraint (6h) denotes the component-wise inequality. The indicator function $1_{\mathbf{d}_{\tau, \omega_{k}} \neq \mathbf{d}_{\tau, \omega_{l}}}$ and vector $\mathbf{d}_{\tau, \omega_{k}}$ are defined as

$$
\begin{aligned}
& 1_{\mathbf{d}_{\tau, \omega_{k}} \neq \mathbf{d}_{\tau, \omega_{l}}}= \begin{cases}1, & \text { if } \mathbf{d}_{\tau, \omega_{k}} \neq \mathbf{d}_{\tau, \omega_{l}}, \\
0, & \text { if } \mathbf{d}_{\tau, \omega_{k}}=\mathbf{d}_{\tau, \omega_{l}},\end{cases} \\
& \mathbf{d}_{\tau, \omega_{k}}=\left(E_{k}^{g}(t+1), \ldots, E_{k}^{g}(\tau)\right), \forall \tau \in \mathcal{T}_{t+1}, \forall k \in \mathcal{K} .
\end{aligned}
$$

Constraints (6b)-(6g) are the extension of constraints in (4b) and (5) under scenario $\omega_{k}$. Constraints (6h) and (6i) are the nonanticipativity constraints [12] of the stochastic optimization. Constraint $(6 \mathrm{~h})$ guarantees that scenarios (i.e., $\omega_{k}$ and $\omega_{l}$ ) with the same available observations at time slot $\tau$ (i.e., $\mathbf{d}_{\tau, \omega_{k}}=\mathbf{d}_{\tau, \omega_{l}}$ ) have the same decisions at time slot $\tau$ (i.e., $\left.\mathbf{e}\left(\tau, \omega_{k}\right)=\mathbf{e}\left(\tau, \omega_{l}\right)\right)$. Note that if $\mathbf{d}_{\tau, \omega_{k}}=\mathbf{d}_{\tau, \omega_{l}}$, we have $1_{\mathbf{d}_{\tau, \omega_{k}} \neq \mathbf{d}_{\tau, \omega_{l}}}=0$ according to (7). Then, we have $\mathbf{e}\left(\tau, \omega_{l}\right) \leq \mathbf{e}\left(\tau, \omega_{k}\right) \leq \mathbf{e}\left(\tau, \omega_{l}\right)$ as in (6h), which is equivalent

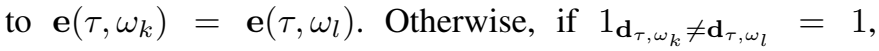

constraint (6h) is always satisfied since each element $\gamma$ in vector $\mathbf{e}^{\max }$ is chosen to be arbitrarily large. Note that the ECS has the same decisions under scenario $\omega_{k}$ and $\omega_{l}$ at time slot $\tau$ if it is unable to distinguish between these scenarios at time slot $\tau$. This case happens if the available observations are the same at time slot $\tau$ because the ECS does not have observations of future time slots. This property is referred to as nonanticipativity, which makes stochastic optimization different from deterministic optimization problems, in which the information of future time slots is assumed to be known. Constraint (6i) guarantees that the ECS has the same decision for the current time slot $t$ under different scenarios, which is due to the nonanticipativiy property [12].

The objective function in problem (6) is piecewise linear since the cost function $c\left(\mathbf{e}\left(\tau, \omega_{k}\right)\right)$ in (2) can be written as

$$
\begin{aligned}
c\left(\mathbf{e}\left(\tau, \omega_{k}\right)\right)=\max & \left\{-p_{s}^{h}(\tau) h-p_{s}^{l}(\tau)\left(E^{x}\left(\tau, \omega_{k}\right)-h\right),\right. \\
& -p_{s}^{h}(\tau) E^{x}\left(\tau, \omega_{k}\right), \\
& \left.p_{e}(\tau)\left(-E^{x}\left(\tau, \omega_{k}\right)\right)\right\} .
\end{aligned}
$$

By introducing auxiliary variables $u\left(\tau, \omega_{k}\right)$, problem (6) can be transformed into a linear program, which can be written as

$$
\begin{aligned}
& \underset{\substack{\mathbf{e}\left(\tau, \omega_{k}\right), u\left(\tau, \omega_{k}\right), \forall \tau \in \mathcal{T}_{t}, \forall k \in \mathcal{K}}}{\operatorname{minimize}} \frac{1}{K} \sum_{k \in \mathcal{K}} \sum_{\tau \in \mathcal{T}_{t}} u\left(\tau, \omega_{k}\right) \\
& \text { subject to } u\left(\tau, \omega_{k}\right) \geq-p_{s}^{h}(\tau) h-p_{s}^{l}(\tau)\left(E^{x}\left(\tau, \omega_{k}\right)-h\right) \text {, } \\
& \forall \tau \in \mathcal{T}_{t}, \forall k \in \mathcal{K}, \\
& u\left(\tau, \omega_{k}\right) \geq-p_{s}^{h}(\tau) E^{x}\left(\tau, \omega_{k}\right), \\
& \forall \tau \in \mathcal{T}_{t}, \forall k \in \mathcal{K}, \\
& u\left(\tau, \omega_{k}\right) \geq p_{e}(\tau)\left(-E^{x}\left(\tau, \omega_{k}\right)\right), \\
& \forall \tau \in \mathcal{T}_{t}, \forall k \in \mathcal{K}, \\
& \text { Constraints }(6 b)-(6 i) \text {. }
\end{aligned}
$$

Note that the linear constraints (10b)-(10d) are included to replace the piecewise linear objective function $c\left(\mathbf{e}\left(t, \omega_{k}\right)\right)$ in (9). Problem (10) is a linear program and can be solved by software such as Matlab optimization toolbox.

\section{Algorithm Description and Complexity}

In this section, we present the energy consumption scheduling algorithm (Algorithm 1) and the computational complexity required to solve problem (10) in Algorithm 1. The algorithm is executed at the beginning of each hour $t$ by the ECS. In Lines 1 and 2, the ECS first initializes the parameters and retrieves the prices for energy consumption and selling from the utility company through the communication network. In Line 3, the ECS constructs scenarios $\boldsymbol{\omega}_{k}, k \in \mathcal{K}$, based on the historical records of the PV output power. For example, the ECS first obtains a forecast of future PV output power. Then, according to the historical statistical forecast error (this can be calculated from the historical forecast results), the ECS generates several different scenarios via Monte-Carlo simulation. In Lines 4-10, the ECS analyzes the scenarios to generate the nonanticipativity constraints according to (6h) and 


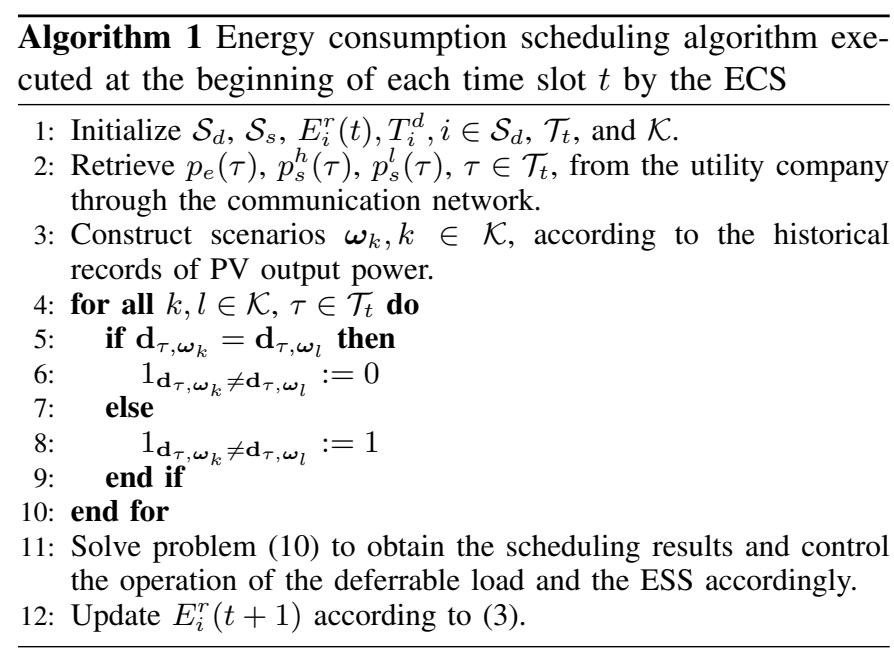

(7). Subsequently, problem (10) is solved and the results are used to control the operation of load and ESS (Line 11). At last, the ECS updates $E_{i}^{r}(t+1)$ to prepare for the scheduling for the next hour (Line 12).

The number of variables considered in problem (10) is $\left(\left|\mathcal{S}_{d}\right|+\left|\mathcal{S}_{s}\right|\right) K\left|\mathcal{T}_{t}\right|$, which is the product of the cardinality of the deferrable load set $\mathcal{S}_{d}$ and the ESS set $\mathcal{S}_{s}$, the number of scenarios considered in the SAA, and the cardinality of set $\mathcal{T}_{t}$ (i.e., the number of operation time slots from the current time slot $t$ onwards). Problem (10) can be solved by the interior point algorithm, which starts from an initial feasible point and approaches the optimal solution iteratively. The average computational complexity bound of the interior point algorithm is $O\left(\left(\left(\left|\mathcal{S}_{d}\right|+\left|\mathcal{S}_{s}\right|\right) K\left|\mathcal{T}_{t}\right|\right)^{3.5} \log \left(\frac{R}{\epsilon}\right)\right)$, where $R$ is the gap between the initial objective value and the optimal value. $\epsilon$ is the gap between the objective value obtained by the interior point algorithm and the optimal value [14].

The number of scenarios $K$ in the SAA technique can be chosen based on the available computation time as well as the accuracy of the PV output power forecast. If the forecast is accurate, we can choose $K$ to be equal to one and use the forecast values to construct scenario $\omega_{1}$. In that case, problem (6) is equivalent to the multi-stage deterministic programming problem. Otherwise, $K$ can be chosen to be either a large value such that problem (10) is equivalent to the nested formulation (4), or a small value to obtain an approximate result with a lower computational complexity.

\section{Performance Evaluation}

In this section, we present simulation results and assess the performance of the proposed energy consumption scheduling algorithm. We compare our proposed algorithm with another algorithm from [3], which we referred to as HDA algorithm. The HDA algorithm is based on real time voltage signal. It activates the idle HVAC load (e.g., water heater) when voltage rise is detected via the sensor. We also compare with the benchmark case where the ECS is not deployed and the deferrable load is not controlled.

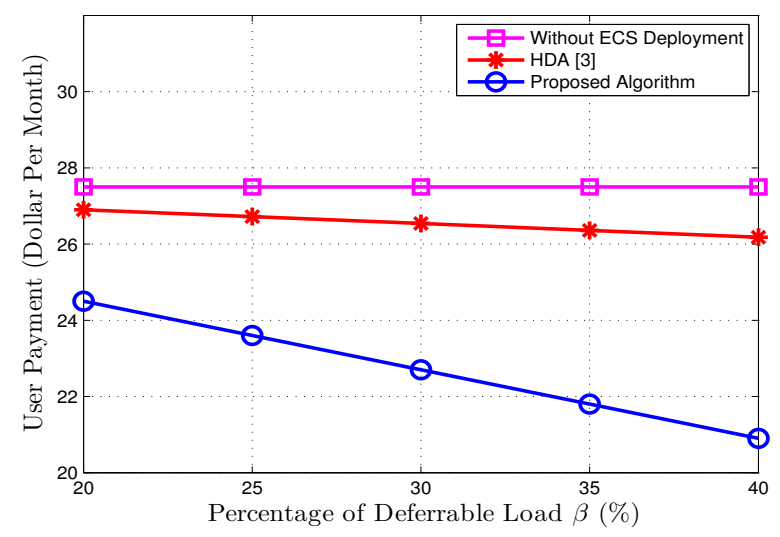

Fig. 4. The monthly electricity bill for a household equipped with PV unit and ESS under different percentage of deferrable load $\beta$. The revenue is calculated under the same pricing scheme.

We consider a distribution network as shown in Fig. 2. There are ten households on each bus and six of the households have installed PV units and ESS. The households have deferrable load, including washing machines, clothes dryers, dish washers, and water heaters. The energy consumption data of the deferrable load is obtained from [5]. The PV output data is taken from [15] and the daily generation is $\sum_{\tau=1}^{24} E^{g}(\tau)=70 \mathrm{kWh}$. The load profile data is obtained from [16] and the household daily energy consumption is $\sum_{\tau=1}^{24}\left(\sum_{i \in \mathcal{S}_{d}} E_{i}^{d}(\tau)+\sum_{j \in S_{m}} E_{j}^{m}(\tau)\right)=120 \mathrm{kWh}$. We denote the percentage of deferrable load by $\beta$. We have

$$
\beta=\frac{\sum_{\tau \in \mathcal{T}} \sum_{i \in \mathcal{S}_{d}} E_{i}^{d}(\tau)}{\sum_{\tau \in \mathcal{T}}\left(\sum_{i \in \mathcal{S}_{d}} E_{i}^{d}(\tau)+\sum_{j \in S_{m}} E_{j}^{m}(\tau)\right)} \times 100 \%
$$

The capacity of the ESS is $2 \mathrm{kWh}$. The maximum charging and discharging power is $0.5 \mathrm{~kW}$ and $-0.5 \mathrm{~kW}$, respectively. We choose $S^{\text {min }}=0.8$. We have $p_{s}^{h}(t)=p_{e}(t)$ and $p_{s}^{l}(t)=$ $0.7 p_{e}(t)$ as our pricing strategy. We set $h=2 \mathrm{kWh}$ as the threshold for IBR pricing. For the distribution network, the power factor of load and PV is 0.92 and 0.95 , respectively. The distance between nearby buses (e.g., bus 1 and bus 2) is 70 feet. The resistance of the power line is $0.8 \mathrm{Ohm} / \mathrm{mile}$. The reactance to resistance ratio is two. The primary feeder base voltage is $12.5 \mathrm{kV}$ [4]. We generate ten scenarios (i.e., $K=10$ ) in the SAA technique.

Fig. 4 shows the household monthly electricity bill versus the percentage of deferrable load. The proposed algorithm helps the household reduce its monthly electricity bill, compared with the case where ECS is not deployed. This is because some of the deferrable load are shifted from the peak hours, when the TOU energy consumption price is relatively high. The proposed algorithm outperforms HDA algorithm with a lower electricity bill as the TOU pricing is not considered in the HDA algorithm. The simulation results also show that the monthly electricity bill decreases as deferrable load percentage increases. This is because more deferrable load can be shifted during peak hours.

We evaluate the performance of our proposed algorithm and HDA algorithm [3] on tackling the voltage rise problem in 


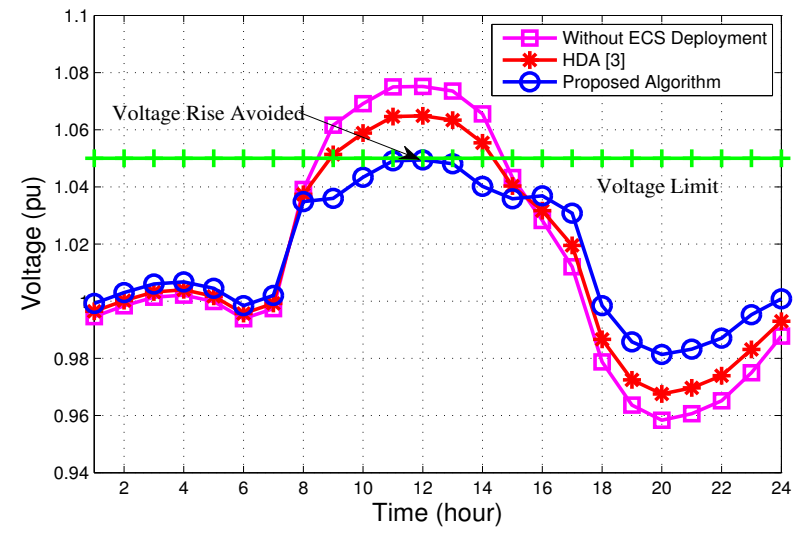

Fig. 5. Comparison of the household voltage profile between the proposed algorithm and HDA algorithm [3]. The voltage is shown in per unit (pu). The base value of pu is the standard voltage (e.g., $120 \mathrm{~V})$. The voltage limit is $1.05 \mathrm{pu}$. We use [4, eqn. (2)] to determine the voltage. The voltage rise is avoided by the proposed algorithm by reducing the reverse energy flow during high solar radiation hours. We set $\beta=30 \%$.

Fig. 5. We use [4, eqn. (2)] to calculate the voltage. Fig. 5 shows the voltage profile at bus 120 in Fig. 2, which has the longest distance from the substation. Note that the voltage rise typically increases with the distance, see [4, eqns. (1) and (2)]. As shown in Fig. 5, our proposed algorithm manages to avoid the voltage rise problem for the households during the noon period (10:00 - 14:00). This is because the households reduce their reverse energy flow during that period by load shifting. The HDA algorithm also reduces the voltage rise but the effect is less than the proposed algorithm. The reason is that the HDA algorithm only controls the HVAC load according to real time voltage feedback signal. Other deferrable loads such as washing machines and dryers are difficult to control using a real time feedback approach.

Fig. 6 shows the peak-to-average ratio (PAR) for the proposed algorithm and HDA algorithm [3] versus the percentage of deferrable load $\beta$. Our proposed algorithm achieves a lower PAR compared with the HDA algorithm. The proposed algorithm encourages households to shift load from the peak hours and reduces the PAR with TOU pricing. The HDA algorithm shifts the HVAC load according to feedback voltage signal and does not include other deferrable loads in its model. In addition, Fig. 6 also shows that when $\beta$ increases, more additional deferrable loads can be shifted to peak hours, which can reduce the PAR of the aggregate load.

\section{CONClusion}

In this paper, we proposed an energy consumption scheduling algorithm to jointly shave peak load and reduce the energy export during high solar radiation hours. We used IBR pricing and TOU pricing to encourage households with PV units and ESS to shift their load from peak hours to high solar radiation hours. A stochastic optimization problem was formulated to minimize the household electricity bill. Simulation results showed the proposed algorithm reduced the electricity bill and the load PAR. It also avoided the voltage rise problem caused by PV integration. For future work, we will study the effect of

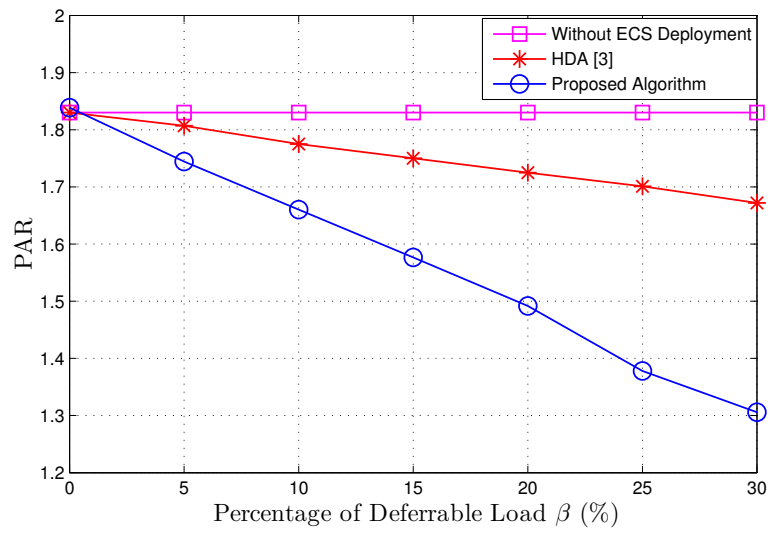

Fig. 6. Comparison of PAR between the proposed algorithm and HDA algorithm [3].

the proposed algorithm on energy loss and battery efficiency.

\section{REFERENCES}

[1] Net Metering in the Illinois State of United States, Available at: www.illinoisattorneygeneral.gov/environment/netmetering.html.

[2] R. A. Walling, R. Saint, R. C. Dugan, J. Burke, and L. A. Kojovic, "Summary of distributed resources impact on power delivery systems," IEEE Trans. on Power Delivery, vol. 23, no. 3, pp. 1636-1644, July 2008.

[3] S. J. Steffel, P. R. Caroselli, A. M. Dinkel, J. Q. Liu, R. N. Sackey, and N. R. Vadhar, "Integrating solar generation on the electric distribution grid," IEEE Trans. on Smart Grid, vol. 3, no. 2, pp. 878-886, June 2012.

[4] X. Liu, A. Aichhorn, L. Liu, and H. Li, "Coordinated control of distributed energy storage system with tap changer transformers for voltage rise mitigation under high photovoltaic penetration," IEEE Trans. on Smart Grid, vol. 3, no. 2, pp. 897-906, June 2012.

[5] A.-H. Mohsenian-Rad, V. W. S. Wong, J. Jatskevich, R. Schober, and A. Leon-Garcia, "Autonomous demand side management based on game-theoretic energy consumption scheduling for the future smart grid," IEEE Trans. on Smart Grid, vol. 1, no. 3, pp. 320-331, Dec. 2010.

[6] N. Li, L. Chen, and S. H. Low, "Optimal demand response based on utility maximization in power networks," in Proc. of IEEE PES General Meeting, Detroit, Michigan, July 2011.

[7] P. Samadi, H. Mohsenian-Rad, R. Schober, and V. W. S. Wong, "Tackling the load uncertainty challenges for energy consumption scheduling in smart grid," IEEE Trans. on Smart Grid, vol. 4, no. 2, pp. 1007-1016, June 2013.

[8] I. Atzeni, L. G. Ordonez, G. Scutari, D. P. Palomar, and J. R. Fonollosa, "Demand-side management via distributed energy generation and storage optimization," IEEE Trans. on Smart Grid, vol. 4, no. 2, pp. 866-876, June 2013.

[9] P. Yang, P. Chavali, and A. Nehorai, "Parallel autonomous optimization of demand response with renewable distributed generators," in Proc. of IEEE SmartGridComm, Tainan, Taiwan, Nov. 2012.

[10] L. Huang, J. Walrand, and K. Ramchandran, "Optimal demand response with energy storage management," in Proc. of IEEE SmartGridComm, Tainan, Taiwan, Nov. 2012.

[11] T. Dang and K. Ringland, "Optimal load scheduling for residential renewable energy integration," in Proc. of IEEE SmartGridComm, Tainan, Taiwan, Nov. 2012.

[12] A. Shapiro, D. Dentcheva, and A. Ruszczynski, Lectures on Stochastic Programming: Modeling and Theory. SIAM, 2009.

[13] N. Sharma, P. Sharma, D. Irwin, and P. Shenoy, "Predicting solar generation from weather forecasts using machine learning," in Proc. of IEEE SmartGridComm, Brussels, Belgium, Oct. 2011.

[14] Y. Ye, Interior-Point Algorithm: Theory and Analysis. John Wiley and Sons, 1997.

[15] Solar Data from National Renewable Energy Laboratory (NREL), Available at: http://www.nrel.gov/solar/.

[16] J. A. Jardini, C. M. V. Tahan, M. R. Gouvea, S. U. Ahn, and F. M. Figueiredo, "Daily load profiles for residential, commercial and industrial low voltage consumers," IEEE Trans. on Power Delivery, vol. 15 no. 1, pp. 375-380, Jan. 2000. 